\title{
Imaging and Histopathology Correlation of Solid Papillary Carcinoma of the Breast : A Review of Four Cases.
}

\author{
Sharifah MIA ${ }^{\mathrm{a}}$, Azwini $M^{\mathrm{a}}$, Nordashima AS \\ aDepartment of Radiology, \\ ${ }^{b}$ Department of Pathology, University Kebangsaan Malaysia, Kuala Lumpur, Malaysia
}

\begin{abstract}
Solid papillary breast carcinoma is a rare type of breast carcinoma. We present four cases which highlight the radiological and histological findings of solid papillary carcinoma. Mammogram supplemented with ultrasound played an important role in detecting solid papillary carcinomas which usually presents as intraductal lesions or intracystic mass with Doppler signal. Excision biopsy is a better option than core biopsy as the latter modality may not be representative and the diagnosis of solid papillary breast carcinoma may be missed.
\end{abstract}

KEYWORDS: Papillary breast neoplasm, breast carcinoma, ultrasound, mammogram.

\section{INTRODUCTION}

Solid papillary carcinoma constitutes only $1-2 \%$ of all breast cancers. It can be divided into non-invasive or invasive carcinomas. ${ }^{1,2}$ The non-invasive type usually presents in older women with palpable mass or bloody nipple discharge. The prognosis of solid papillary breast carcinoma is more favourable than other ductal carcinoma and reportedly presents with less nodal involvement. ${ }^{3}$ Invasive solid papillary carcinoma usually has a higher incidence of lymph node involvement and has a poor prognosis.

\section{CASE REPORT}

Case 1

A 55-year-old post-menopausal lady presented with a left breast lump. She had 4 children, all of whom she breast fed. Other risk factor included being on hormone replacement therapy for the past one year. There was no family history of breast or ovarian carcinoma. On examination, there was a $1.5 \mathrm{~cm}$ firm lesion at left 12 o'clock position. Mammogram and ultrasound showed bilateral breast cysts. Fine needle aspiration cytology revealed no evidence of malignancy. Six months down

Corresponding author:

Dr Sharifah Majedah Idrus Alhabshi

Radiologist and Senior Lecturer

Department of Radiology,

University Kebangsaan Malaysia Medical Centre,

Jalan Yaacob Latif, Bandar Tun Razak,

56000 Cheras, Kuala Lumpur, Malaysia.

Telephone: +603-91455555

Fax: +603-9145 6682

Email: shmajedah@yahoo.com the line she presented again with enlarging left 12 o'clock breast lump.

Mammogram showed moderately dense lesion with irregular margin and complementary ultrasound revealed a large heterogenous mass with mixed cystic and solid component at left 12 o'clock position measuring $2.9 \times 2.8 \mathrm{~cm}$ with internal vascularity within. There were also bilateral breast cysts. A left axillary lymphadenopathy with thickened cortex was also noted. A diagnosis of highly suspicious breast carcinoma with BI-RADS 5 classification was reported.

Ultrasound guided core biopsy was done and subsequently this lady underwent a left wide local excision. Histological examination revealed a well circumscribed tumour comprised of closely apposed arborizing and anastomosing papillae with fine fibrovascular core (Figure 1). The tumour cells morphology was consistent with solid papillary carcinoma, grade II (based on Bloom and Richardson grading system).

The nearest margin was $2 \mathrm{~mm}$ away from the tumour. Immunohistochemical study showed a complete negativity of p63 compatible with the diagnosis of solid papillary carcinoma. There were absence of estrogen receptor (ER), progesterone receptor (PR) and neuroendocrine expression. Six months mammogram and ultrasound surveillance did not show evidence of recurrence. 


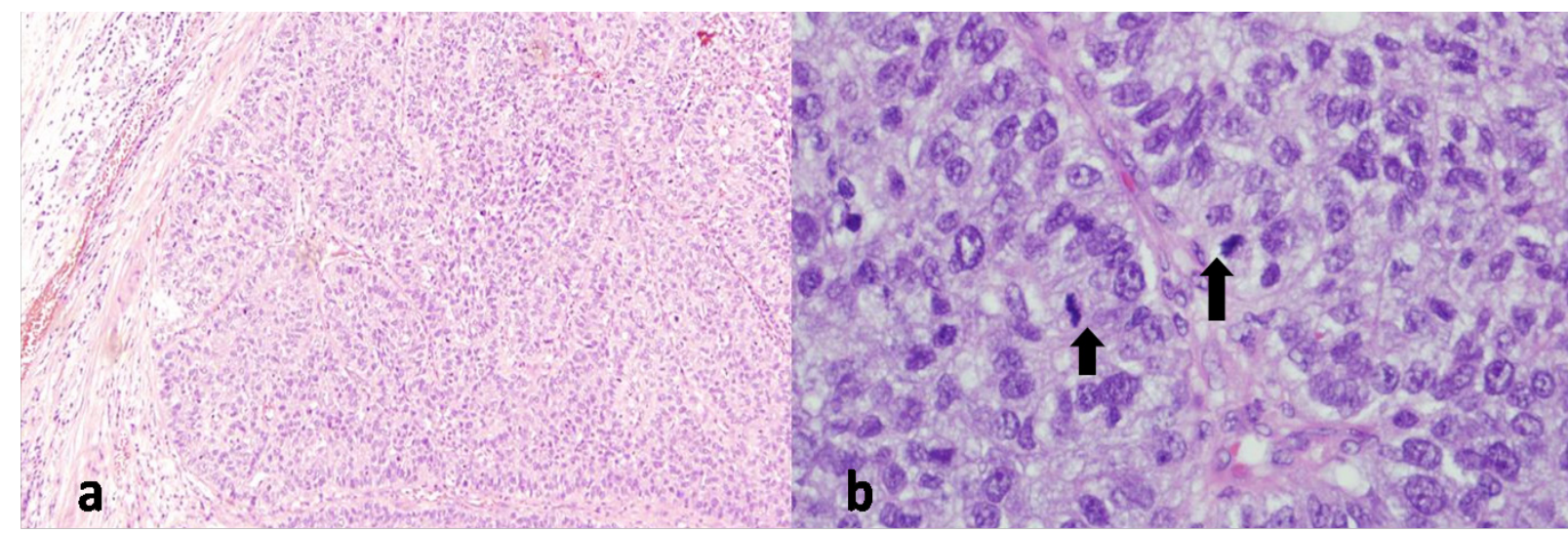

Figure 1: (a) Circumscribed, expansile cellular mass comprised of closely apposed papillae(H\&E,x100) (b) Photomicrograph shows papillae lined by tumour cells. Mitotic figures are seen (arrows). (H\&E,x400).

\section{Case 2}

A 61-year-old post-menopausal lady presented with an incidental findings of left breast lump during screening. She breast-fed all her three children and had no family history of breast carcinoma. She had been on hormone replacement therapy for the last six months. A mammogram done showed a lobulated lesion in the left upper outer quadrant. An ultrasound showed two intraductal lesions at left 3 o'clock position with mix cystic and solid component. Ultrasound guided core

biopsy was done and subsequently left wide local excision and sentinel node biopsy were performed. Histological examination revealed a solid papillary carcinoma with mucinous component, grade II (BloomRichardson grading) and lymph node involvement (Figure 2). The estrogen (ER) and progesterone (PR) receptors status were positive. Patient then underwent axillary dissection, chemotheraphy and radiotherapy. She was on hormonal therapy as the ER and PR status were positive.

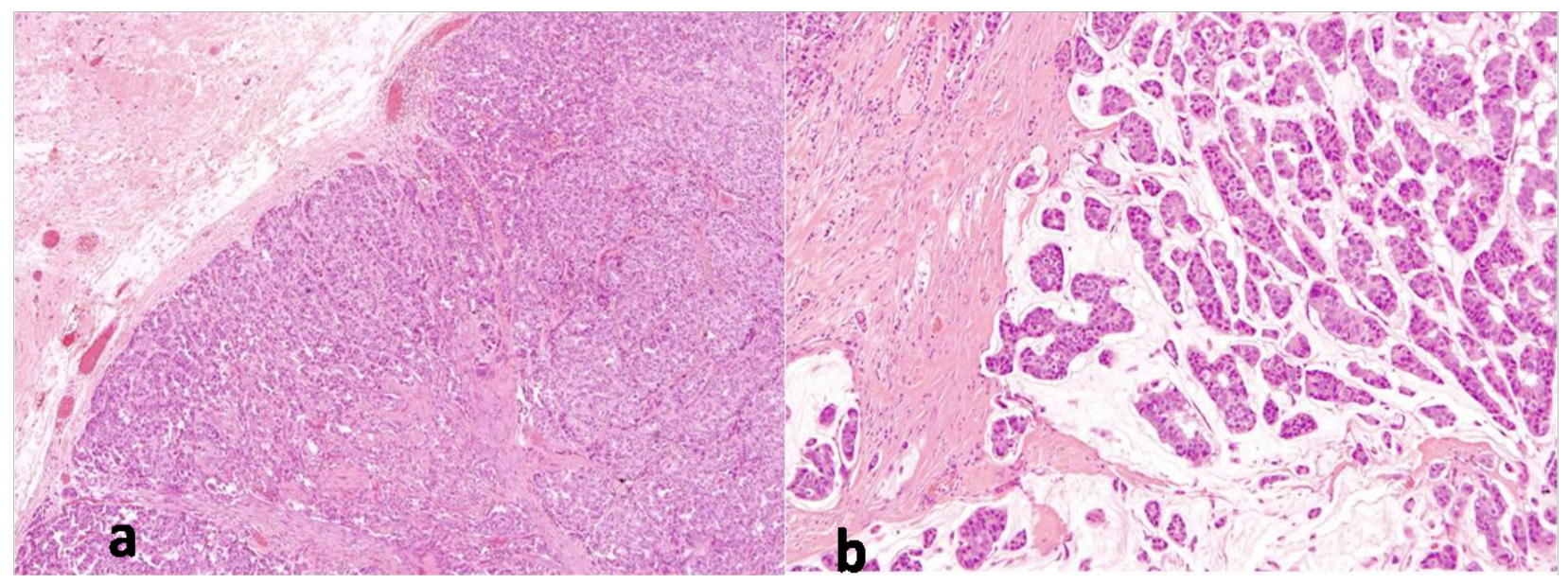

Figure 2: (a) Well circumscribed cellular tumour comprised of closely apposed papillae. Extracellular mucin is discernible between epithelial cells (left lower part of field). (H\&E, X40)

(b) Clusters of malignant cells in pools of extracellular mucin. (H\&E, x100)

\section{Case 3}

A 52-year-old woman with incidental finding of a left breast nodule on routine mammogram screening. There was no family history of breast carcinoma and no other risk factors. Mammogram revealed a welldefined moderately dense mass in the left periareolar region with several smaller lesions in the upper outer quadrant, associated with clusters of amorphous microcalcifications (Figure 3a). Complementary ultrasound showed well defined, heterogenous internal echo lesions with mix solid cystic component and several satellite lesions at left periareolar region (Figure $3 \mathrm{~b}$ \& $3 \mathrm{c}$ ). Ultrasound guided core biopsy was done and histological exmination revealed papillary neoplasm which was highly suggestive of papillary ductal carcinoma in-situ. Subsequently, wide local excision and sentinel node biopsy were performed. The definitive histological examination confirmed a diagnosis of intracystic papillary carcinoma with multiple foci of high grade ductal carcinoma in-situ (DCIS). Immunohistochemical study showed strong expressions of estrogen and progesterone receptors. Patient currently had completed four cycles of chemotherapy and was planned for radiotherapy. 


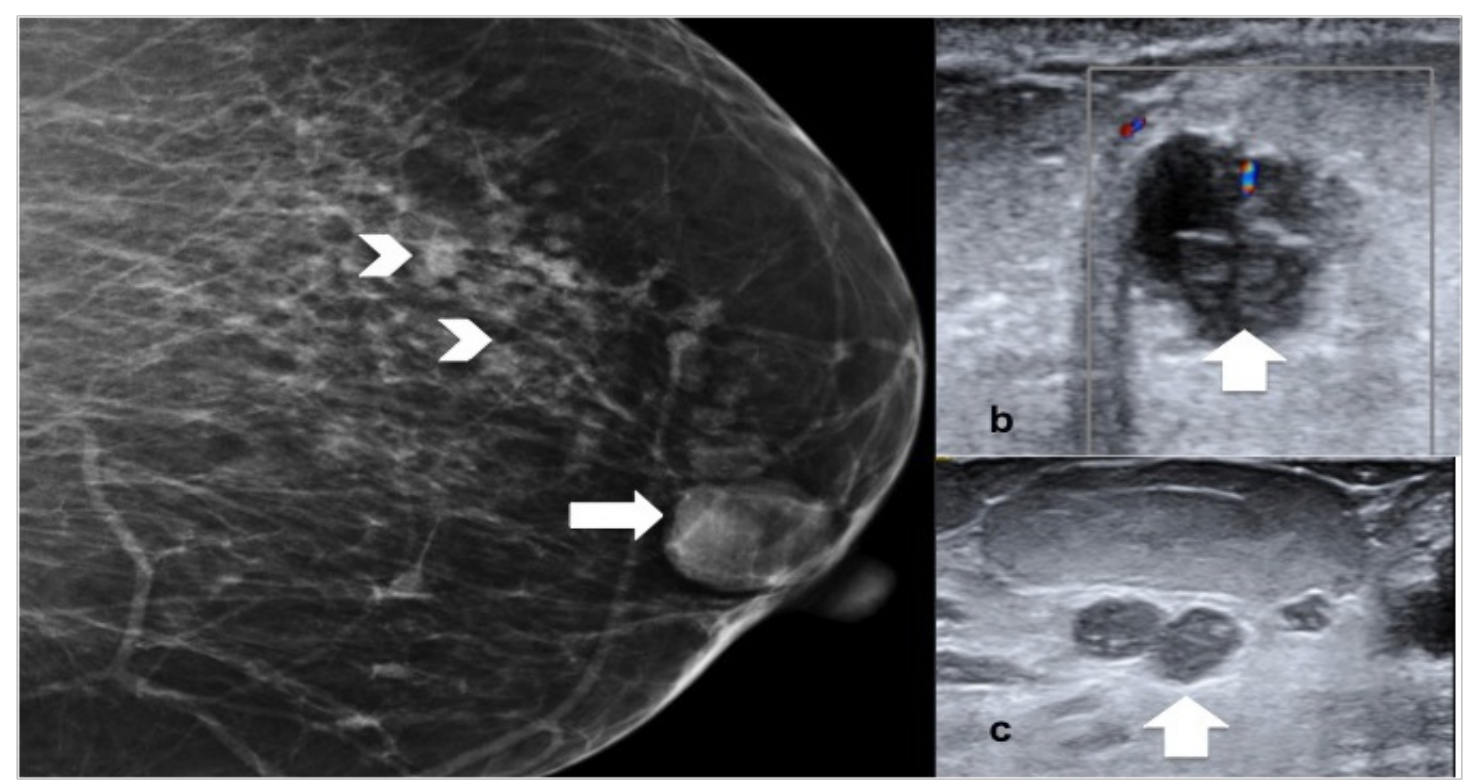

Figure 3: A 52-year-old woman with incidental findings of left breast lesions. Mammogram craniocaudal view showing a well defined moderately dense mass at the left periareolar lesion (arrow) with cluster of microcalcification at upper outer quadrant and several satellite lesion at upper-midquadrant (arrow head). (a) Ultrasound showing well defined masses at the left upper outer quadrant with heterogenous internal echo and mix solid and cystic component within (c) and interal vascularity demonstrated on Doppler study (b).

\section{Case 4}

A 35-year-old nulliparous woman presented with a six months history of right breast lump. There was no family history of breast carcinoma. On examination, there was a $3 \times 3 \mathrm{~cm}$ mobile hard lump at the right lower outer quadrant. Ultrasound revealed a huge well defined lobulated heterogenous internal echo mass at right 7 o'clock position with mix solid and cystic component. Some of the solid component showed papillary projection (Figure 4). Proceeded with ultrasound guided core biopsy. During biopsy, thick brownish fluid continuously came out from the biopsy

site. Histological features of the biopsy were consistent with papillary carcinoma with mucinous component or mucinous carcinoma. A wide local excision and sentinel node biopsy was performed. Histological examination confirmed a diagnosis of an encapsulated papillary carcinoma. There was no associated in-situ or invasive component. Immunohistochemical study showed absence of estrogen receptor (ER) and progesterone receptor (PR) expressions. There was no lymph node involvement.

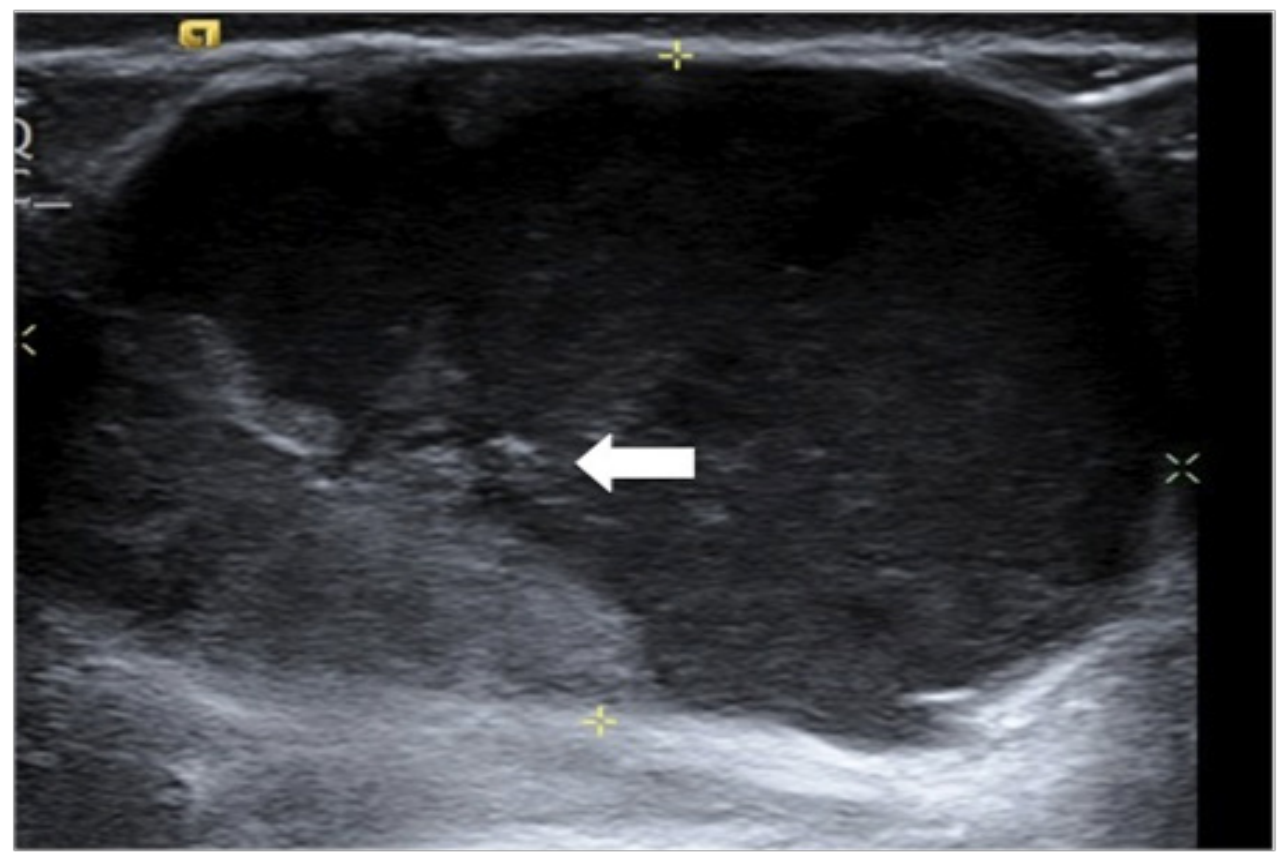

Figure 4: A 35-year-old nulliparous woman presented with right breast mass. Ultrasound of the right breast at 7 o'clock position showing a large well defined lobulated heterogenous internal echo mas with mix solid and cystic component. Papillary projections are also seen arising from the solid component (arrow). 


\section{DISCUSSION}

Intraductal solid papillary carcinoma is a rare form of breast carcinoma with varied clinical and radiological presentations as demonstrated from the cases described. It can be divided into invasive and noninvasive types.

We present four cases of the non-invasive solid papillary carcinoma of the breast. Solid papillary carcinoma generally occurs in older women aged 63 to 67 years. Three of our patients are elderly with more than 50 years of age and one patient is 35 years old. Patients with solid papillary carcinoma may present with a palpable mass or bloody nipple discharge. The tumour may also be asymptomatic and identified at screening mammography. All of our patients present either with clinically palpable mass or an incidental finding during screening. None of our patients present with nipple discharge.

Solid papillary carcinoma has a slow growth pattern and has better prognosis than other forms of ductal carcinomas. ${ }^{3}$ Axillary nodal metastases are infrequent. Only one of our patients showed evidence of micro metastases of axillary node. None of the patients had distance metastases at presentation.

Three basic patterns of intraductal solid papillary carcinoma are recognized on ultrasound: intraductal mass with or without ductal dilatation, intracystic mass and a predominantly solid pattern with the intraductal mass totally filling the duct. Benign papillomas are also known to exhibit microcalcifications. Intracystic solid papillary carcinomas are highly vascular tumours and have a propensity to bleed spontaneously. A distinct vascular pedicle is identified within the central core of intrapapillary neoplasm, with branching vessels arborising within the mass. ${ }^{4}$ This is the most important ultrasound features to differentiate cyst with gravity dependant debris from intracystic solid papillary neoplasm.

Mammogrammayonoccasions be unable to differentiate between simple cyst and papillary neoplasm because these lesions usually demonstrate a well defined solitary round, oval or lobulated mass or clusters of well-defined masses with low to moderate density mass on mammogram. Therefore supplementary ultrasound is mandatory to differentiate between simple cyst and intracystic solid papillary neoplasm and color Doppler sonography may show intratumoral blood flow or large feeding vessels. ${ }^{1}$

Occasionally, there will be either satellite nodules and or microcalcifications as presented in one of our case whereby the largest lesion confirmed to be solid papillary carcinoma and the smaller intraductal satellite lesions with clusters of microcalcification were high grade ductal carcinoma in situ.

Benign papillomas and solid papillary carcinomas have similar overlapping features on ultrasound, therefore it may not be possible to differentiate these lesions via ultrasound. However, in the older older age group with a larger solid component and intracystic doppler signal, solid papillary carcinomas are more likely.

Pathologically, papillary carcinoma has a frond-forming growth pattern supported by a fibrovascular stalk. The absence of a myoepithelial layer distinguishes carcinoma from benign papillary lesion. The tumour is classified as papillary ductal carcinoma in-situ if the epithelium of a papillary carcinoma has features diagnostic of intraductal component. If a cystic component is present, the tumour is described as an intracystic papillary carcinoma. Invasive solid papillary carcinoma occurs infrequently, often as only a small focus of stromal invasion and is almost always detected at the periphery of the lesion. ${ }^{2}$ Intracystic solid papillary carcinoma also can occur in a pure form, or it may be associated with ductal carcinoma in situ or invasive carcinoma not otherwise specified..$^{1,5}$

Differentiation between in-situ and invasive solid papillary carcinoma is also difficult by fine needle aspiration or core biopsy because the centre solid part of the lesion is often targeted during the procedure but features of invasion is often found at the periphery of the tumour. Therefore, excisional biopsy should be performed for a small intraductal and intracystic lesion if clinically suspicious of papillary carcinoma. ${ }^{2,6}$

Intracystic papillary carcinoma is usually managed by wide local excision rather than mastectomy due to its less aggressive nature and good prognosis. Radiation therapy may also be administered. Sentinel lymph node biopsies or axillary dissections are often performed to assess the axillary lymph nodes. The prognosis for intracystic solid papillary carcinoma is usually very good with disease-specific survival rates approaching $100 \%$. Encapsulated papillary carcinoma can be treated with adequate local radiation therapy. Routine use of adjuvant therapy, particularly chemotherapy, is not clearly appropriate in view of the very low risk and good prognosis unlike invasive ductal carcinoma. However, hormonal therapy may be indicated in certain cases such as papillary carcinoma that show evidence of estrogen and progesteron receptor status positivity. ${ }^{1}$ Solid papillary carcinoma generally expressed ER and neuroendocrine differentiation. Our first patient presented with an unusual presentation of solid papillary carcinoma with negative hormonal receptors.

\section{CONCLUSION}

Our case reports together with previous reported cases of solid papillary carcinoma have demonstrated that mammogram has limitations in the diagnosis and the use of ultrasound supplements the role of imaging in detecting solid papillary carcinomas which usually presents as intraductal lesions or intracystic mass with Doppler signal. The clinical and radiological findings can be subtle and easily missed at times. It may not be 
possible to differentiate benign from malignant lesions via imaging alone, however, a high index of suspicion in the presence of certain characteristics may favour malignant lesions. Excision biopsy is a better option than core biopsy or fine needle aspiration cytology as the later may not be representative and the diagnosis of solid papillary breast carcinoma may be missed.

\section{REFERENCES}

1. Soo MS, Williford ME, Walsh R, Bentley RC, Kornguth P. Papillary carcinoma of the breast: imaging findings. AJR Am J Roentgenol 1995; 164:321-6.

2. Muttarak M, Lerttumnongtum $P$, Chaiwun B, Peh WC. Spectrum of papillary lesions of the breast: clinical, imaging and pathologic correlation. AJR Am J Roentgenol 2008; 191:700-707.

3. Ibarra JA. Papillary lesions of the breast. Breast J 2006; 12:237-51.

4. Ganesan S, Karthik G, Joshi M, Damodaran V. Ultrasound spectrum in intraductal papillary neoplasms of breast. British Journal of Radiology 2006; 79:843-9.

5. Ibarra JA. Papillary lesions of the breast. Breast J 2006; 12:237-51.

6. GunhanBilgen I, Zekioglu O, Ustun EE, Memis A \& Erhan Y. Invasive micropapillary carcinoma of the breast: clinical, mammographic, and sonographic findings with histopathologic correlation. AJR Am J Roentgenol 2002; 179:92731. 